\title{
A novel de novo $B R C A 1$ mutation in a Chinese woman with early onset breast cancer
}

\author{
Ava Kwong • Enders K. O. Ng • Edmund Y. H. Tang • Chris L. P. Wong • \\ Fian B. F. Law $\cdot$ Candy P. H. Leung $\cdot$ Aaron Chan $\cdot$ M. T. Cheung $\cdot$ \\ M. Y. To $\cdot$ Edmond S. K. Ma $\cdot$ Dee W. West $\cdot$ James M. Ford
}

Published online: 15 March 2011

(c) The Author(s) 2011. This article is published with open access at Springerlink.com

\begin{abstract}
Germline mutations in the two breast cancer susceptibility genes, BRCAl and BRCA2 account for a significant portion of hereditary breast/ovarian cancer. De novo mutations such as multiple exon deletion are rarely occurred in BRCA1 and BRCA2. During our mutation screening for $B R C A 1 / 2$ genes to Chinese women with risk factors for hereditary breast/ovarian cancer, we identified a novel germline mutation, consisting of a deletion from exons 1 to 12 in BRCAl gene, in a patient diagnosed with early onset triple negative breast cancer with no family history of cancer. None of her parents carried the mutation
\end{abstract}

Oral presentation in part at the 16th Hong Kong International Cancer Congress November 4-6, 2009 and publication only presentation in part at ASCO meeting May 29-June 2, 2009, Abstract No: e22143.

Electronic supplementary material The online version of this article (doi:10.1007/s10689-011-9429-y) contains supplementary material, which is available to authorized users.

A. Kwong $(\varangle) \cdot$ E. K. O. Ng · E. Y. H. Tang

C. P. H. Leung - A. Chan

Division of Breast Surgery, Queen Mary and Tung Wah Hospital, The University of Hong Kong, Pokfulam, Hong Kong e-mail: akwong@asiabreastregistry.com

A. Kwong · E. K. O. Ng · C. L. P. Wong ·

F. B. F. Law - M. T. Cheung - M. Y. To - E. S. K. Ma Department of Molecular Pathology and Department of Surgery,

Hong Kong Sanatorium and Hospital, Happy Valley, Hong Kong

D. W. West

Cancer Prevention Institute of California, Fremont, CA, USA

A. Kwong · D. W. West · J. M. Ford

Stanford University School of Medicine, Stanford, CA, USA

A. Kwong

The Hong Kong Hereditary Breast Cancer Family Registry,

Happy Valley, Hong Kong and molecular analysis showed that this novel de novo germline mutation resulted in down-regulation of BRCAI gene expression.

Keywords Breast cancer - BRCAl gene ·

De novo mutation - Early onset

$\begin{array}{ll}\text { Abbreviations } \\ \text { DCIS } & \text { Ductal carcinoma in-situ } \\ \text { TN } & \text { Triple negative cancer } \\ \text { ER } & \text { Estrogen receptor } \\ \text { BIC } & \text { Breast cancer information core } \\ \text { qRT-PCR } & \text { Quantitative reverse transcription-polymerase } \\ & \text { chain reaction }\end{array}$

\section{Introduction}

Breast cancer is the most frequently occurring malignancy in women. Germline mutations in the two breast cancer susceptibility genes, BRCA1 and BRCA2 account for a significant portion of hereditary breast/ovarian cancer [1]. BRCA mutations are distributed over all exons and therefore genetic testing for these BRCA1/2 genes require a mutation screening method of the entire coding regions of the genes. In our laboratory in Hong Kong, we currently offer mutation screening for BRCA1/2 genes to Chinese women with breast or ovarian cancer at age 50 years and younger, or with a family history of BRCA related cancers such as breast, ovarian, colon, and prostate, using direct full gene sequencing. However, in many countries, breast cancer patients without family history are generally not tested. For example, in the United Kingdom it is estimated that up to $6 \%$ of the BRCA1 and BRCA2 mutation cases are 
missed because they lack a family history of relevant cancers. Lack of a family history may relate to small family size, non-penetrance, premature death, loss of contact with family members, and inadequate information [2]. Alternatively, lack of family history can also be explained by new germline mutations found in the probands but not in any of their family members. Such de novo mutations have been reported in diseases such as hemophilia A [3] and thalassaemia [4]. De novo mutations are very rare among $B R C A$ genes [5-8]. Moreover, most of the reported diseaseassociated mutations in BRCA1 and BRCA2 result in frame shift, nonsense, insertions, deletions, or splice site alterations, which lead to formation of a truncated BRCA protein. However, large genomic rearrangements in $B R C A 1$ and BRCA2 are rarely seen, and a similar BRCA1 deletion (from exon 1 to 13) has been reported in Finland [9]. Hereby, we describe a de novo mutation in which multiple exons were deleted from BRCAl in a Chinese breast cancer patient. Furthermore, we demonstrate that significant reduction in BRCAl RNA levels in the proband by qRT-PCR assays, suggesting the absence of truncated BRCAI RNA products. The results from this case report illustrate the importance of gene rearrangement testing in addition to full gene sequencing in detecting $B R C A 1$ and $B R C A 2$ mutations in breast or ovarian cancer patients, even in the absence of a strong family history.

\section{Materials and methods}

Patient history and paternity confirmation

We report a 33-year old Chinese woman who at age 30 was diagnosed with grade 2 invasive ductal NOS breast carcinoma and confirmed to be a triple negative cancer (ER, PR and Her2/Neu negative). No family history of cancers was reported. Due to a complicated family history (Fig. 1), familial relationship of this group was confirmed by paternity test (Identifiler ${ }^{\mathrm{TM}}$ System, 15+1 markers, Applied Biosystems Foster City, CA). The results of paternity test were shown in Supplementary Tables 1-3.

\section{Conventional DNA sequencing}

BRCA1 and BRCA2 mutation detection was performed on genomic DNA extracted from peripheral blood samples or paraffin embedded tissue. DNA was extracted using QIAGEN DNA Mini Kit (Qiagen, Hilden, Germany) according to manufacturer instructions. Mutation analysis was performed by direct DNA sequencing of all coding exons of $B R C A 1$ and $B R C A 2$ and partial flanking intronic sequences as described previously [10]. PCR conditions and primer sequences are available upon request. Bi-directional

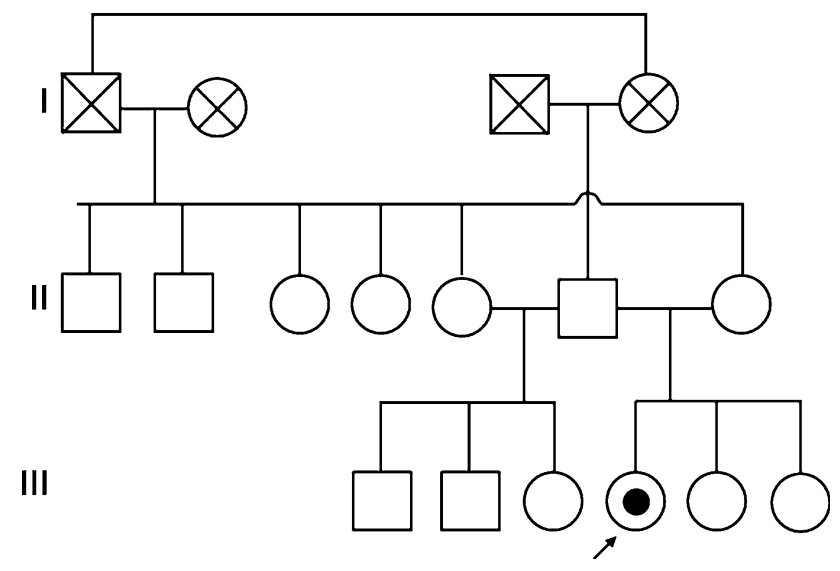

Fig. 1 Family pedigree of proband family. The proband (III-4) is indicated by an arrow

sequencing was performed using BigDye Terminator v3.1 Cycle Sequencing Kit and analyzed on an ABI 3130xl genetic analyzer (Applied Biosystems). Sequencing results were compared with the reference DNA sequences (NM_007294.3) using Variant Reporter software (Applied Biosystems) and then reviewed manually. Computational analysis for potential cryptic splice site mutation was performed using splice site prediction programs such as NNSPLICE and ESEF finder when sequence changes were identified. All mutation and sequence variants are named according to the recommendations for the description of sequence variants of Human Genome Variation Society (HGVS).

Multiplex ligation-dependent probe amplification

Multiplex Ligation-dependent Probe Amplification (MLPA) was performed to determine the presence of large genomic rearrangements in the DNA of the proband. The MLPA kit P002 and P045 (MRC-Holland, Amsterdam, Netherlands) was used for BRCA1 and BRCA2 gene copy number determination respectively [10-14]. DNA samples were processed according to manufacturer protocol. Each MLPA analysis was carried out with 5 normal control samples. Amplification products were electrophoresed on an ABI 3130xl genetic analyzer and interpreted using GeneMapper software (Applied Biosystems). Results were exported to the Coffalyser program (MRC-Holland) for further analysis.

RNA extraction and quantitative RT-PCR

Total RNA was extracted from blood samples of patients and controls. $0.5 \mu \mathrm{g}$ of total RNA was reversely transcribed into cDNA with High Capacity cDNA Reverse Transcription kit (Applied Biosystems). Real-time qPCR was 
performed using QuantiTect SYBR Green PCR Kit (Qiagen) in ABI PRISM 7900 HT system (Applied Biosystems). All primer sequences are listed in Supplementary Table 4. The expression levels were normalized to housekeeping genes, Glyceraldehyde 3-phosphate dehydrogenase $(G A P D H) . \triangle \mathrm{Ct}$ was calculated by subtracting the $\mathrm{Ct}$ values of $G A P D H$ genes from the $\mathrm{Ct}$ values of BRCAl. $\Delta \Delta \mathrm{Ct}$ was then calculated by subtracting $\Delta \mathrm{Ct}$ of the proband from $\Delta \mathrm{Ct}$ of non-proband. Fold change of gene was calculated by the equation $2^{-\Delta \Delta C t}$.

\section{Statistical analysis}

Statistical significance was determined by Mann-Whitney test. All $P$-values are two-sided and $<0.05$ was considered statistically significant by GraphPad PRISM 4 software (GraphPad Software, La Jolla, CA).

\section{Results}

Based on the lower incidence of breast cancer in Asia cohorts $[10,15]$, we offer $B R C A 1 / 2$ genetic testing for clinically high risk female patients; high risk being defined as having (1) at least one first- or second-degree relative with breast and/or ovarian cancer, regardless of age; (2) a diagnosis made when less than 51 years of age; (3) bilateral breast cancer; (4) triple negative or medullary type pathology; (5) at least one relative with cancers other than breast and ovarian cancer that are known to be related to $B R C A$ mutations; or (6) ovarian cancer with a family history of breast cancer. Between March 2007 to November
2010, a total of 530 Chinese female patients with breast and/or ovarian cancer were screened by $B R C A 1 / 2$ genetic testing. Genomic DNA extracted from peripheral blood samples were sent to Hong Kong Hereditary Breast Cancer Family Registry. BRCA1 and BRCA2 mutation screening was performed by direct sequencing of all exons in BRCA1 and $B R C A 2$ and partial flanking intronic sequences $[10,16$, 17]. Our sequencing results identified 68 (12.8\%) deleterious $B R C A$ gene mutations. A total of 29 (29/530, 5.5\%) were in BRCA1 and $39(39 / 530,7.4 \%)$ in BRCA2. During this period of $B R C A 1 / 2$ genetic testing, this 33 year old Chinese patient was also eligible for such genetic testing. Based on our sequencing results, nucleotide changes in both $B R C A 1$ and $B R C A 2$ from the proband were listed in Supplementary Table 5. In addition to DNA sequencing, MLPA was used to determine if large genomic rearrangements existed for the proband. As a result, this is the only case so far we identified a large deletion of exon 1 to exon 12 of BRCA1 by MLPA (Fig. 2). Analysis of BRCA2 did not show any signs of large deletions or duplications. MLPA was also conducted on 5 family members of the proband: mother, father, biological sister, maternal aunt and half sister (daughter of proband's maternal aunt and the same biological father) (Fig. 1). No signs of this or other large deletions in either $B R C A 1$ or $B R C A 2$ in any of the family members were detected, suggesting that there are no carriers of the reported large deletion in the family. Therefore the reported large deletion appears to be a de novo mutation.

To investigate whether the defective $B R C A 1$ allele in the proband could produce protein product, we first attempted to perform RT-PCR to detect whether the presence of
Fig. 2 MLPA probes signals and result of the analysis. Coffalyser Sample Plate Generator (S.P.G.) was used to automatically create sample plate files for MLPA analysis. Results shown that probe signals from exon 1 to 12 were significantly decreased indicating the presence of deletion across those exons

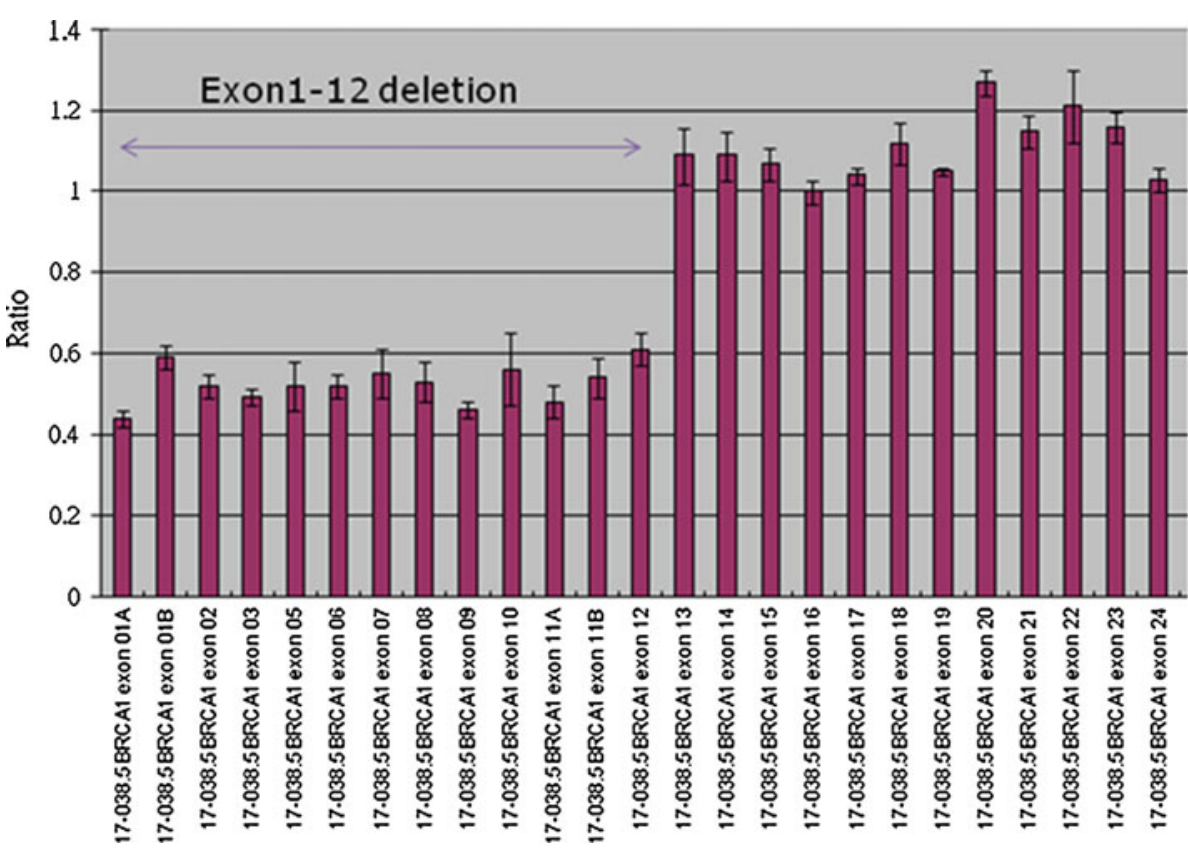


truncated BRCAl RNA products for protein truncation testing, but our attempts were not successful. Therefore, we postulate such truncated RNA product is not expressed in the proband. To prove our postulation that such truncated $B R C A 1$ RNA product do not exist, one of the indirect ways is to compare BRCAl RNA levels between proband and non-proband. If the amount of BRCAl RNA levels expressed from both wild-type and defective alleles in the proband are significantly lower than control, it would be reasonable to conclude the absence of the truncated RNA products from the defective allele. Thus, we need to design four sets of qRT-PCR primer pairs in which two sets of primer pairs targeting region within the putative deletion (PCR amplicons cover exon 2-7 and exon 10-11, respectively). Thus, these qPCR assays only amplify BRCAl RNA from wild-type allele). The other 2 set of primer pairs target the region outside the putative deletion (PCR amplicon cover exon 17-20 and exon 23-24, respectively). Therefore these qPCR assays amplify BRCAl RNA from both wildtype and deleted alleles. As a result, BRCA1 RNA levels from each amplicon region (within or outside deleted regions) were compared between proband and non-proband (non-BRCA mutation carriers) including family members $(n=5)$ and normal controls $(n=18)$. Our results indicated that for RT-PCR targeting the deleted region (either E2-7 or E10-11), BRCA1 RNA levels in the proband were significantly lower than that in non-probands (All $P$-values $<0.05$, Mann-Whitney test; Fig. 3a). Similarly, for RT-PCR targeting outside the deleted region (either E17-20 or E23-24), BRCAI RNA levels in the proband were also significantly lower than that in non-probands (All $P$-values $<0.05$, Mann-Whitney test; Fig. 3b). Combining all this data, it demonstrates that overall BRCAl RNA expression levels in the proband (defective allele carrier) was significantly lower than that in controls, suggesting the existence of truncated $B R C A 1$ RNA products is unlikely.

\section{Discussion}

In this study, we report a novel de novo BRCAl mutation encompassing deletion from exons 1 to 12 in a Chinese breast cancer patient of early onset with no family history. Such de novo mutation is extremely rare and has not been reported so far. As the large deletion was identified only by MLPA, it underlines the importance of pursuing gene rearrangement testing if full gene sequencing fails to detect mutations or deletions in BRCA1/2 genes. Furthermore, significant reduction in BRCAl RNA expression levels in the proband is confirmed by RT-PCR assays targeting both PCR products within and outside deleted region, suggesting no truncated BRCAl RNA transcript was expressed. Thus, our unsuccessful attempts to amplify the suspected
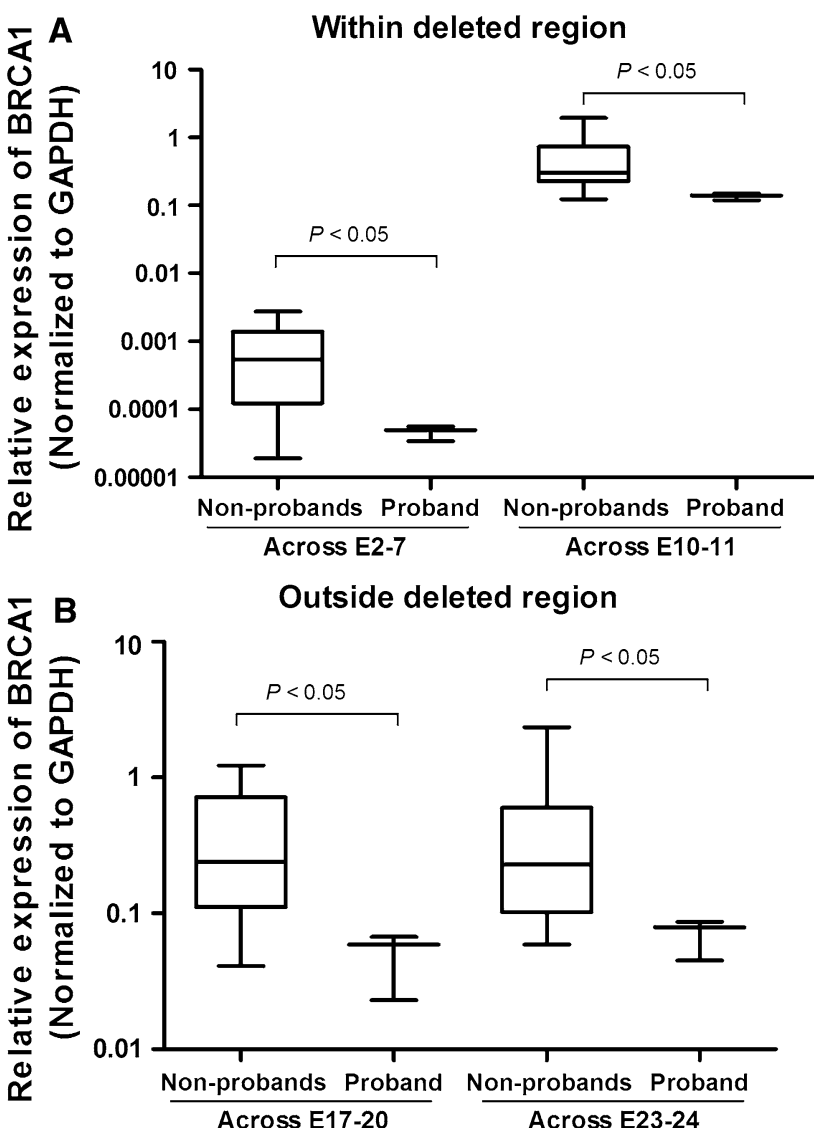

Fig. 3 BRCA1 RNA level comparison between proband and nonprobands (family members and normal controls). Box plots of BRCAI RNA expression levels a targeting the deleted region b targeting outside the deleted region of the proband and non-proband $(n=23)$. Expression levels ( $\log 10$ scale at $Y$-axis) are normalized to GAPDH. The lines inside the boxes denote the medians. The boxes mark the interval between the 25 th and 75 th percentiles. The whiskers denote the interval between the 10th and 90th percentiles. Statistically significant differences were determined using Mann-Whitney tests

truncated product for protein truncation test were expected. Such deletion results in suppression of BRCA1 protein production in the defective allele. Unfortunately, the breakpoint location of the deletion is still being mapped. We postulate that the deletion breakpoint is located more than $10 \mathrm{~kb}$ upstream from the BRCAl transcriptional start site.

Although most of the identified deleterious mutations in $B R C A 1 / 2$ are truncating mutations resulting in a nonfunctional protein, large gene rearrangements related to deletion may not be detected by non-quantitative techniques [18]. The emerging technologies such as the use of high density probes combined with array comparative genomic hybridization $(\mathrm{aGCH})$ has identified novel large genomic deletions [19]. Mutation screening is important in early onset breast cancer patients even if there is no family history.

Many factors might lead to the observed mutation in the studied family. This could be due to sporadic germline 
BRCAl mutations, or the mutation could have been inherited from an asymptomatic mosaic parent. Consanguineous marriage in this family could be another factor. The rate of consanguineous marriages in Asian countries such as China, Singapore and Hong Kong was below $10 \%$ [20]. This was lower than Iran in which the rate is around $40 \%$ and the first cousin marriages are the most common type can affect the pattern of genetic disorders in the population [21]. Another report estimated that 20\% of cases of breast cancer diagnosed in women in Pakistan aged below 40 years are attributable to first-cousin marriages [22].

In conclusion, we report a novel de novo mutation in the BRCAl gene, resulting in breast cancer in a very young Chinese woman, consisting of a deletion from exon 1 to 12 . Such de novo mutation is extremely rare and to the best of our knowledge this is the first case reported in Asian population so far. This patient presents significantly low expression levels of $B R C A l$ RNA, indicating the existence of truncated $B R C A 1$ RNA products is unlikely.

Acknowledgments We sincerely thank to Dr Ellen Li Charitable Foundation and Kerry Group Kuok Foundation for their support. We also like to thank Dr Dacita Suen, Dr Catherine Choi and Ms. Wong Ling for helping in patient recruitment.

Open Access This article is distributed under the terms of the Creative Commons Attribution Noncommercial License which permits any noncommercial use, distribution, and reproduction in any medium, provided the original author(s) and source are credited.

\section{References}

1. Ford D, Easton DF, Stratton M et al (1998) Genetic heterogeneity and penetrance analysis of the BRCA1 and BRCA2 genes in breast cancer families. The Breast Cancer Linkage Consortium. Am J Hum Genet 62(3):676-689. doi:S0002-9297(07)63848-8

2. Edwards E, Yearwood C, Sillibourne J, Baralle D, Eccles D (2009) Identification of a de novo BRCA1 mutation in a woman with early onset bilateral breast cancer. Fam Cancer 8(4):479-482. doi: 10.1007/s10689-009-9270-8

3. Antonarakis SE, Kazazian HH, Tuddenham EG (1995) Molecular etiology of factor VIII deficiency in hemophilia A. Hum Mutat 5(1):1-22. doi:10.1002/humu.1380050102

4. Rojnuckarin P, Settapiboon R, Vanichsetakul P, Sueblinvong T, Sutcharitchan P (2007) Severe beta(0) thalassemia/hemoglobin E disease caused by de novo 22-base pair duplication in the paternal allele of beta globin gene. Am J Hematol 82(7):663-665. doi: 10.1002/ajh.20816

5. Robson M, Scheuer L, Nafa K, Ellis N, Offit K (2002) Unique de novo mutation of BRCA2 in a woman with early onset breast cancer. J Med Genet 39(2):126-128
6. Tesoriero A, Andersen C, Southey M et al (1999) De novo BRCA1 mutation in a patient with breast cancer and an inherited BRCA2 mutation. Am J Hum Genet 65(2):567-569. doi:10.1086/ 302503

7. van der Luijt RB, van Zon PH, Jansen RP, van der Sijs-Bos CJ, Warlam-Rodenhuis CC, Ausems MG (2001) De novo recurrent germline mutation of the BRCA2 gene in a patient with early onset breast cancer. J Med Genet 38(2):102-105

8. Hansen TV, Bisgaard ML, Jonson L et al (2008) Novel de novo BRCA2 mutation in a patient with a family history of breast cancer. BMC Med Genet 9:58. doi:10.1186/1471-2350-9-58

9. Pylkas K, Erkko H, Nikkila J, Solyom S, Winqvist R (2008) Analysis of large deletions in BRCA1, BRCA2 and PALB2 genes in Finnish breast and ovarian cancer families. BMC Cancer 8:146. doi:10.1186/1471-2407-8-146

10. Kwong A, Wong LP, Wong HN et al (2009) A BRCA2 founder mutation and seven novel deleterious BRCA mutations in southern Chinese women with breast and ovarian cancer. Breast Cancer Res Treat 117(3):683-686. doi:10.1007/s10549-0090385-2

11. Sellner LN, Taylor GR (2004) MLPA and MAPH: new techniques for detection of gene deletions. Hum Mutat 23(5):413-419

12. Hogervorst FB, Nederlof PM, Gille JJ et al (2003) Large genomic deletions and duplications in the BRCA1 gene identified by a novel quantitative method. Cancer Res 63(7):1449-1453

13. Schouten JP, McElgunn CJ, Waaijer R, Zwijnenburg D, Diepvens F, Pals G (2002) Relative quantification of 40 nucleic acid sequences by multiplex ligation-dependent probe amplification. Nucleic Acids Res 30(12):e57

14. Bunyan DJ, Eccles DM, Sillibourne J et al (2004) Dosage analysis of cancer predisposition genes by multiplex ligation-dependent probe amplification. Br J Cancer 91(6):1155-1159

15. Kwong A, Wong CH, Shea C, Suen DT, Choi CL (2010) Choice of management of southern chinese BRCA mutation carriers. World J Surg. doi:10.1007/s00268-010-0477-5

16. Kwong A, Wong LP, Chan KY, Ma ES, Khoo US, Ford JM (2008) Characterization of the pathogenic mechanism of a novel BRCA2 variant in a Chinese family. Fam Cancer 7(2):125-133. doi:10.1007/s10689-007-9155-7

17. Kwong A, Ng EK, Law FB et al (2010) High-resolution melting analysis for rapid screening of BRCA2 founder mutations in Southern Chinese breast cancer patients. Breast Cancer Res Treat. doi:10.1007/s10549-010-0882-3

18. Walsh T, Casadei S, Coats KH et al (2006) Spectrum of mutations in BRCA1, BRCA2, CHEK2, and TP53 in families at high risk of breast cancer. JAMA 295(12):1379-1388. doi:10.1001/jama. 295.12.1379

19. Staaf J, Torngren T, Rambech E et al (2008) Detection and precise mapping of germline rearrangements in BRCA1, BRCA2, MSH2, and MLH1 using zoom-in array comparative genomic hybridization (aCGH). Hum Mutat 29(4):555-564. doi:10.1002/ humu. 20678

20. Bildirici M, Kokdener M, Ersin O (2010) An empirical analysis of the effects of consanguineous marriages on economic development. J Fam Hist 35(4):368-394

21. Saadat $M$ (2007) Consanguineous marriages in Iranian folktales. Community Genet 10(1):38-40. doi:10.1159/000096280

22. Liede A, Malik IA, Aziz Z, Rios Pd Pde L, Kwan E, Narod SA (2002) Contribution of BRCA1 and BRCA2 mutations to breast and ovarian cancer in Pakistan. Am J Hum Genet 71(3):595-606. doi:S0002-9297(07)60339-5 